\title{
Energy-dependent hot-spots model via vector meson photoproduction
}

\section{Krelina*}

Universidad Tecnica Federico Santa Maria \& CCTVAL, Casilla 110, Valparaiso, Chile; Czech Technical University in Prague, FNSPE, Brehova 7, 11519 Prague, Czech Republic

E-mail: michal.krelina@usm.cl

\section{J. Cepila, J.G. Contreras}

Czech Technical University in Prague, FNSPE, Brehova 7, 11519 Prague, Czech Republic

\section{Tapia Takaki}

The University of Kansas, Dept. of Physics and Astronomy, Lawrence, KS, USA

\begin{abstract}
We present a model for the QCD structure of hadrons as seen in the dipole picture. The model is based on hot spots - regions of large gluonic density - populating the impact parameter space. In our model, the number of hot spots grows with energy and their positions fluctuate event-by-event. Using this model, we calculate coherent and incoherent photoproduction of vector mesons (VM) off a proton and nuclear targets. We compare our predictions with current data from HERA, RHIC and the LHC at different energies. We also present new signatures of saturation effects that could be observed with current and future data.
\end{abstract}

XIII Quark Confinement and the Hadron Spectrum - Confinement2018

31 July - 6 August 2018

Maynooth University, Ireland

\footnotetext{
${ }^{*}$ Speaker.
} 


\section{Introduction}

Diffractive photoproduction of vector mesons in high-energy interactions is a sensitive probe of the gluon distribution of hadrons in the small- $x$ region, where saturation effects are expected to be important. One of the most standard way to calculate the exclusive and dissociative vector mesons photoproduction is using the Good-Walker formalism [1]. In this formalism, the dissociative photoproduction is calculated as a variance of the amplitude and, therefore, this model is event-by-event fluctuations sensitive.

In high energy physics, it is well known that the number of gluons increases with energy or decreasing Bjorken $x$. And, at a certain value, the number of gluons is saturated, i.e., they are in a region where the probability of gluon fusion is high.

Assuming the Good-Walker formalism and a hot-spot model, where the number of hot spots increases with energy, similarly as gluons do, it is possible to study saturation effects as was done for the first time in [2]. Since the dissociative photoproduction is calculated as the variance, one can see a decreasing cross section at very high energies. This is because of the high number of hot spots when the transverse profile of the proton is starting to look as a uniform distribution leading to zero variance.

This formalism can be generalized to the nuclear case, as was done in [3], and used for predictions for UPC measurement at the LHC and RHIC and, for example, for EIC predictions. Moreover, taken into consideration other vector mesons with different masses, one can find the saturation scale at different energies as was demonstrated in [4].

\section{Exclusive and inclusive VM production}

The cross sections for the photoproduction of a vector meson VM off a target $\mathrm{T}$ for a photon (transverse (T) or longitudinal (L) polarization) have the forms

$$
\left.\frac{d \sigma(\gamma T \rightarrow V M T)}{d t}\right|_{T, L}=\frac{\left(R_{g}^{T, L}\right)^{2}}{16 \pi}\left|\left\langle\mathscr{A}\left(x, Q^{2}, \vec{\Delta}\right)_{T, L}\right\rangle\right|^{2}
$$

for the exclusive or coherent processes, and

$$
\left.\frac{d \sigma(\gamma T \rightarrow V M Y)}{d t}\right|_{T, L}=\frac{\left(R_{g}^{T, L}\right)^{2}}{16 \pi}\left(\left\langle\left|\mathscr{A}\left(x, Q^{2}, \vec{\Delta}\right)_{T, L}\right|\right\rangle^{2}-\left|\left\langle\mathscr{A}\left(x, Q^{2}, \vec{\Delta}\right)_{T, L}\right\rangle\right|^{2}\right)
$$

for dissociative or incoherent production, where $Y$ represents the dissociative state. $R_{g}^{T, L}$ is the skewedness correction, and $\vec{\Delta}^{2}=-t$. The amplitude is given by

$$
\mathscr{A}\left(x, Q^{2}, \vec{\Delta}\right)_{T, L}=i \int d^{2} r \int_{0}^{1} \frac{d z}{4 \pi}\left(\Psi^{*} \Psi_{V M}\right)_{T, L} \int d^{b} e^{-i(\vec{b}-(1-z) \vec{r}) \cdot \vec{\Delta}} \frac{d \sigma_{d p / A}}{d^{2} b}
$$

where $\Psi_{V M}$ is the wave function of the vector meson, $\Psi$ is the wave function of a virtual photon fluctuating into a quark-antiquark color dipole, $r$ is the transverse distance between the quark and the antiquark, and $z$ is the fraction of the longitudinal momentum of the dipole carried by the quark. For more details see $[2,3,4]$. 
For proton the dipole cross section was modeled as

$$
\frac{d \sigma_{d p}}{d^{2} b}=\sigma_{0} N(x, r) T_{p}(\vec{b})
$$

and for nuclear target two models were used [3]

$$
\frac{d \sigma_{d A}^{G G}}{d^{2} b}=2\left(1-e^{-\frac{1}{2} \sigma_{0} N(x, r) T_{p}(\vec{b})}\right), \quad \frac{d \sigma_{d A}^{G S}}{d^{2} b}=\sigma_{0}^{A}\left(1-e^{-\frac{1}{4} r^{2} Q_{A, s}^{2}(x)}\right) T_{A}(\vec{b}) .
$$

In both cases, the fluctuations are implemented in the profile functions $T_{p / A}$. For the proton we use

$$
T_{p}(\vec{b})=\frac{1}{N_{h s}} \sum_{i=1}^{N_{h s}} T_{h s}\left(\vec{b}-\vec{b}_{i}\right), \quad T_{h s}\left(\vec{b}-\vec{b}_{i}\right)=\frac{1}{2 \pi B_{h s}} e^{-\frac{\left(\vec{b}-\vec{b}_{i}\right)^{2}}{2 B_{h s}}}
$$

where the position of a hot spot (hs) is a random point extracted from a 2D-Gaussian distribution centered at zero and of width $B_{P}$ related to the size of the proton. For the nuclear case, we sample the position of nucleons using random points from a Wood-Saxon distribution where each nucleon has a hot-spot structure as just described. $N_{h s}$ is a random number drawn from a zero-truncated Poisson distribution, where the Poisson distribution has a mean value [3]

$$
\left\langle N_{h s}(x)\right\rangle=p_{0} x^{p_{1}}\left(1+p_{2} \sqrt{x}\right) .
$$

\section{Results}

Figure 1 (left) shows the energy dependence of the cross section for the dissociative production of a vector meson off a proton as a function of $W_{p}$ for $\rho^{0}, J / \psi$ and $\Upsilon(1 S)$. The dissociative cross section presents a maximum after which there is a steep descent for $J / \psi$ and the same behavior can be seen also for $\rho^{0}$ and $\Upsilon(1 \mathrm{~S})$. The position of the maximum is correlated with the mass of the vector meson.

Figure 1 (right) shows the cross section for the exclusive production of a vector meson off a proton as a function of $W_{p}$ for different vector mesons. The predictions of the model are compared to the available data from HERA and the LHC. The description of data using our model is quite satisfactory in the whole domain.

For the nuclear case, we study the coherent and incoherent photoproduction of $\rho^{0}$ off the following nuclei: $\mathrm{Xe}, \mathrm{Au}$, and $\mathrm{Pb}$. The nuclei are modeled with Woods-Saxon distributions. The deformation of the Xenon nucleus is taken into account parameterising the radius of the nucleus as a function of the polar angle. The results of our model for $\rho^{0}$ coherent photoproduction off a nucleus (A) at a photon-nucleus centre-of-mass energy $W_{A}$ are shown in Fig. 2 for the GG-hs, GG-n and GS-hs cases. Note, "hs" denotes geometry of using the hot spots, and "n" denotes assuming only nucleons fluctuations.

Figures 3 show coherent (left) and incoherent (right) photoproduction cross section prediction for Xe-Xe collisions measured during the Run II at the LHC. 

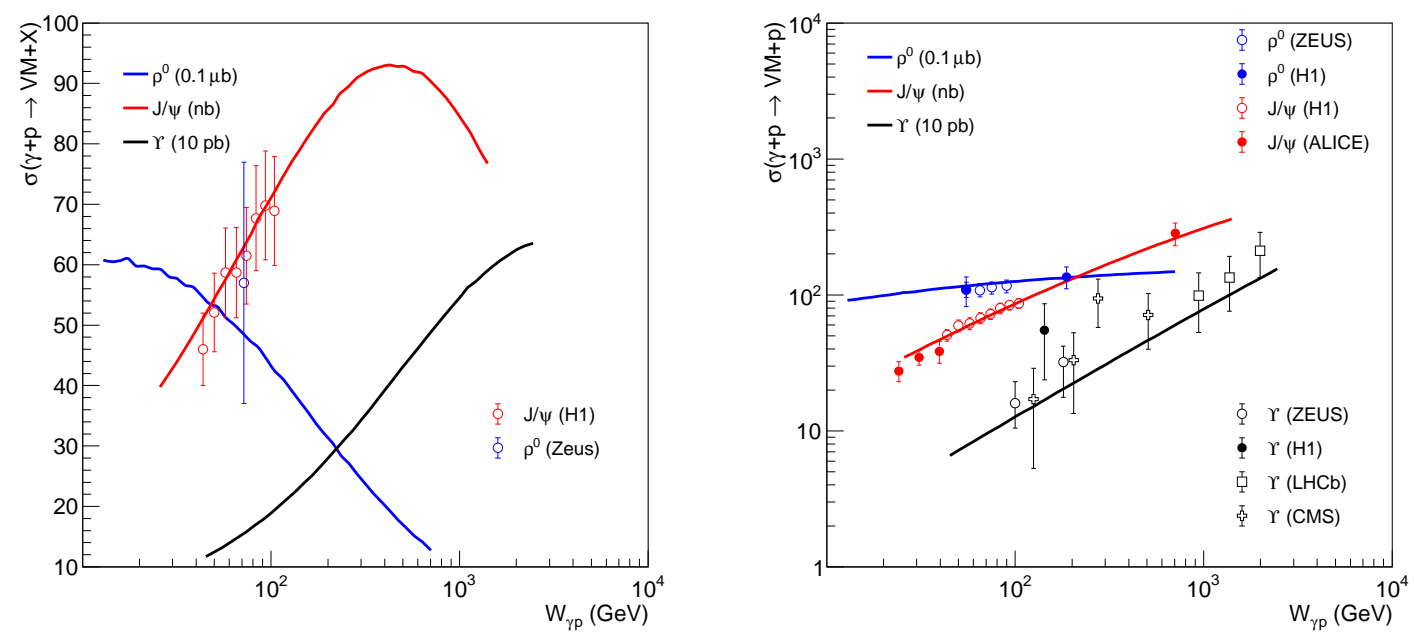

Figure 1: Energy dependence for $\rho^{0}, J / \psi$, and $\Upsilon(1 S)$ dissociative (left) and exclusive (right) photoproduction off protons as predicted by our model (solid lines) compared to measurements [5, 6] of dissociative and $[7,8,9,10,11]$ of exclusive protoproduction. Note that the different cross sections are displayed in different units.

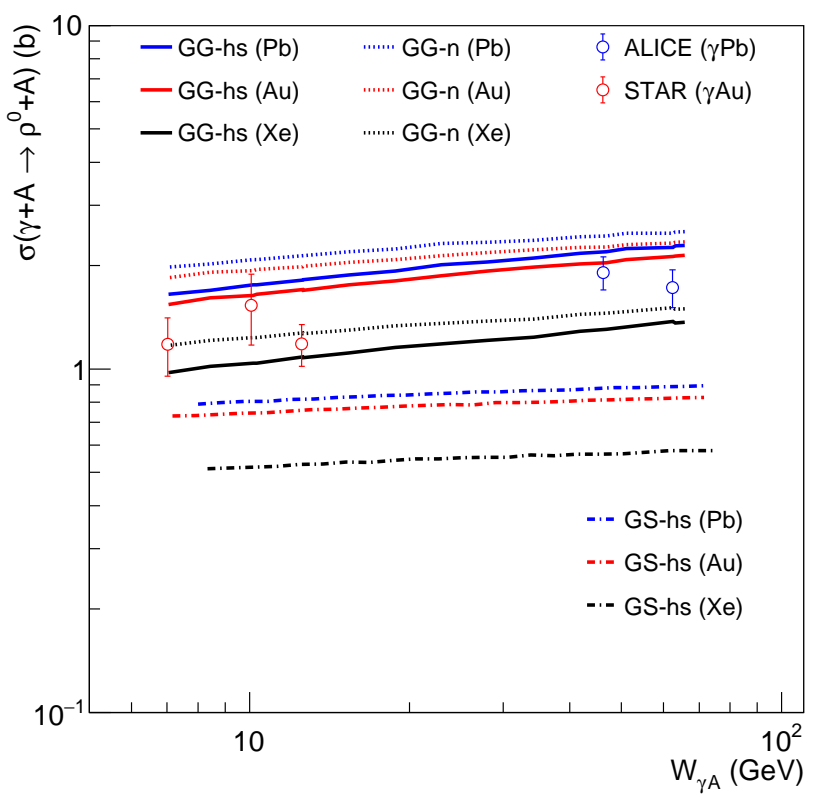

Figure 2: Energy dependence for $\rho^{0}$ coherent photoproduction off a nucleus (A) as predicted by our model. The approach based on geometric-scaling ideas, GS, is shown with dashed- dotted lines, while the GlauberGribov, GG, approach is shown with solid (dotted) lines for the calculation including hot spots (only nucleons). The model is compared to measurements $[12,13,14,15,16]$ 

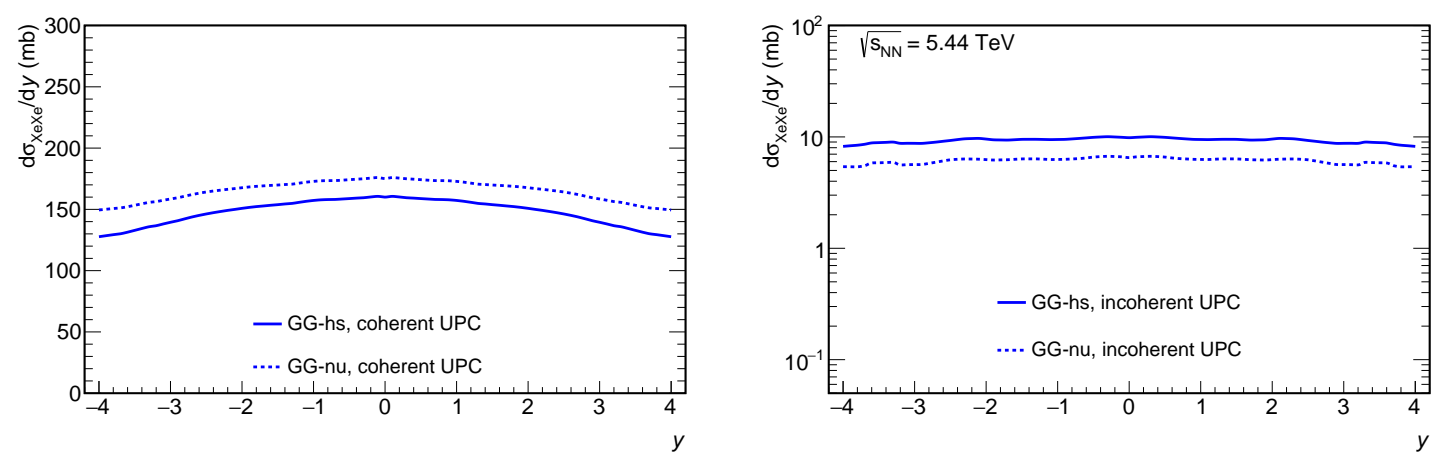

Figure 3: Prediction of the cross section for coherent (left) and incoherent (right) photonuclear $\rho^{0}$ production in Xe-Xe collisions at $\sqrt{s_{N N}}=5.44 \mathrm{TeV}$ using the Glauber-Gribov, GG, approach.

\section{Conclusions}

The model predicts for $J / \psi$ that the energy dependence of the dissociative process increases from low energies up to $W_{\gamma p} \sim 500 \mathrm{GeV}$ and then decreases steeply - this energy range can be explored at the LHC.

The physics explanation according to the parton saturation phenomenon is that the growth of the number of scattering centers provides the increase of the exclusive and dissociative cross section. However, at some point, the number of hot spots is so large that they overlap. When the overlap is large enough, different configurations look the same, and the variance diminishes, and so does the dissociative cross section.

The mass and scale dependence of vector meson production provides a new handle in the search for saturation effects - this can be checked experimentally by re-processing data from HERA.

The effects of the energy-dependent hot-spots model can be seen for the nuclear targets in an energy dependence of the ratio of the incoherent to coherent cross sections. This prediction can be tested with already existing data from RHIC and the LHC Run 2.

\section{Acknowledgements}

The work of Michal Krelina was supported in part by Fondecyt grant Postdoctorado N.3180085 (Chile), by the grant 17-04505S of the Czech Science Foundation (GACR) (CZ) and partly this work has been performed in the framework of COST Action CA15213 THOR (EU). Access to computing and storage facilities of the National Grid Infrastructure MetaCentrum provided under the programme CESNET LM2015042 of the Czech Republic is greatly appreciated.

\section{References}

[1] M. L. Good and W. D. Walker, Phys. Rev. 120, 1857 (1960).

[2] J. Cepila, J. G. Contreras and J. D. Tapia Takaki, Phys. Lett. B 766, 186 (2017) [arXiv:1608.07559 [hep-ph]]. 
[3] J. Cepila, J. G. Contreras and M. Krelina, Phys. Rev. C 97, no. 2, 024901 (2018) [arXiv:1711.01855 [hep-ph]].

[4] J. Cepila, J. G. Contreras, M. Krelina and J. D. Tapia Takaki, Nucl. Phys. B 934, 330 (2018) [arXiv:1804.05508 [hep-ph]].

[5] C. Alexa et al. [H1 Collaboration], Eur. Phys. J. C 73, no. 6, 2466 (2013) [arXiv:1304.5162 [hep-ex]].

[6] J. Breitweg et al. [ZEUS Collaboration], Eur. Phys. J. C 2, 247 (1998) [hep-ex/9712020].

[7] S. Aid et al. [H1 Collaboration], Nucl. Phys. B 463, 3 (1996) [hep-ex/9601004].

[8] C. Adloff et al. [H1 Collaboration], Phys. Lett. B 483, 23 (2000) [hep-ex/0003020].

[9] S. Chekanov et al. [ZEUS Collaboration], Phys. Lett. B 680, 4 (2009) [arXiv:0903.4205 [hep-ex]].

[10] B. B. Abelev et al. [ALICE Collaboration], Phys. Rev. Lett. 113, no. 23, 232504 (2014) [arXiv:1406.7819 [nucl-ex]].

[11] R. Aaij et al. [LHCb Collaboration], JHEP 1509, 084 (2015) [arXiv:1505.08139 [hep-ex]].

[12] C. Adler et al. [STAR Collaboration], Phys. Rev. Lett. 89, 272302 (2002) [nucl-ex/0206004].

[13] B. I. Abelev et al. [STAR Collaboration], Phys. Rev. C 77, 034910 (2008) [arXiv:0712.3320 [nucl-ex]].

[14] G. Agakishiev et al. [STAR Collaboration], Phys. Rev. C 85, 014910 (2012) [arXiv:1107.4630 [nucl-ex]].

[15] J. Adam et al. [ALICE Collaboration], JHEP 1509, 095 (2015) [arXiv:1503.09177 [nucl-ex]].

[16] V. Pozdniakov et al. [ALICE Collaboration], PoS EPS -HEP2017, 186 (2017) [arXiv:1710.03417 [hep-ex]]. 